\title{
Glioblastoma Circulating Cells: Reality, Trap or Illusion?
}

\author{
A. Lombard, ${ }^{1,2}$ N. Goffart, ${ }^{1}$ and B. Rogister ${ }^{1,3,4}$ \\ ${ }^{1}$ Laboratory of Developmental Neurobiology, GIGA-Neuroscience, University of Liège, Liège, Belgium \\ ${ }^{2}$ Department of Neurosurgery, $\mathrm{CHU}$ and University of Liège, Liège, Belgium \\ ${ }^{3}$ Department of Neurology, CHU and University of Liège, Liège, Belgium \\ ${ }^{4}$ GIGA-Development, Stem Cells and Regenerative Medicine, University of Liège, Liège, Belgium
}

Correspondence should be addressed to B. Rogister; bernard.rogister@ulg.ac.be

Received 3 March 2015; Accepted 19 April 2015

Academic Editor: Yupo Ma

Copyright (C) 2015 A. Lombard et al. This is an open access article distributed under the Creative Commons Attribution License, which permits unrestricted use, distribution, and reproduction in any medium, provided the original work is properly cited.

\begin{abstract}
Metastases are the hallmark of cancer. This event is in direct relationship with the ability of cancer cells to leave the tumor mass and travel long distances within the bloodstream and/or lymphatic vessels. Glioblastoma multiforme (GBM), the most frequent primary brain neoplasm, is mainly characterized by a dismal prognosis. The usual fatal issue for GBM patients is a consequence of local recurrence that is observed most of the time without any distant metastases. However, it has recently been documented that GBM cells could be isolated from the bloodstream in several studies. This observation raises the question of the possible involvement of glioblastoma-circulating cells in GBM deadly recurrence by a "homing metastasis" process. Therefore, we think it is important to review the already known molecular mechanisms underlying circulating tumor cells (CTC) specific properties, emphasizing their epithelial to mesenchymal transition (EMT) abilities and their possible involvement in tumor initiation. The idea is here to review these mechanisms and speculate on how relevant they could be applied in the forthcoming battles against GBM.
\end{abstract}

\section{Introduction}

Circulating tumor cells (CTC) are the main required substrate for cancer to spread and extend metastases. These cells originally come from the primary tumor and reach the vascular compartment. CTC are then able to leave the circulation, migrate through the conjunctive tissue of different organs, and proliferate to form metastases. It remains unclear whether CTC are able to go back to the primary tumor site, specifically after therapeutic treatment, and therefore to participate to tumor recurrence.

In fact, it has been suggested that a very small proportion of CTC can form metastases. This subpopulation of cells is called circulating tumor stem cells (CTSC). Indeed, this subpopulation is thought to be self-renewing, multipotent, and capable of tumor initiation [1]. Up to now, different hypotheses try to explain their presence in the peripheral blood, involving several mechanisms to cross the vascular barrier. Because of their properties, these cells are of high interest to counteract the evolution of the disease and metastases formation. This review aims to better understand the biology of these CTSC with a particular focus on glioblastoma multiforme, a grade IV malignant brain tumor characterized by a dead-end prognosis, systematic relapses, and rare metastases.

\section{Origins, Circulation, and Destinations of Circulating Tumor Stem Cells (CTSC)}

CTC come from the initial tumor or from eventual metastases. In the tumor mass, less than $5 \%$ of malignant cells [2] are known to preserve a self-renewal potential through multiple generations and are able to create a new tumor. These are called cancer stem cells (CSC). Classically, CSC are defined by three major in vitro properties: formation of spherical colonies in culture suspension, differential levels and patterns of surface markers, and increased survival after radiation or chemotherapeutic treatment [3-7]. Moreover, in experimental models, those CSC are the only tumor cells able to initiate the development of new tumors in heterotopic or homotopic xenotransplantation experiments. These CSC present high tolerance to the lethal environment, host defense and growth-suppression factors thanks to immune 
mediators, cell cycle checkpoints, and DNA damage control pathways [8].

From this, different hypotheses attempted to elucidate the presence of CSC in the blood or circulating tumor stem cells (CTSC). CSC can use a normal morphogenetic process called Epithelial Mesenchymal Transition (EMT) [9] to modify their features in order to escape the tissue of origin and to migrate towards the vascular compartment [10]. Liu and collaborators recently demonstrated that differentiated tumor cells acquire migratory abilities due to the development of EMT pathways [11] (Figure 1(a)). The intravasation is finally possible by the secretion of enzymes, such as serine/cysteine proteases, matrix metalloproteases (MMP) or disintegrins, and other metalloproteases (ADAMS), in order to degrade the basal membrane of blood vessels [12]. The presence of tumorassociated macrophages (TAMs), especially in hypoxic region of tumor [13], seems indeed to facilitate the intravasation process, maybe via secretion of MMP-9 [14].

Once in the bloodstream, most of the CTC, including CSC, undergo an important selection by shear forces or natural killer (NK) cells from the immune system [15]. However, CTC can aggregate to cellular elements [16] or platelets [17] and express several receptor tyrosine kinases (RTK), antiapoptotic molecules and invasion signaling components $[16,18]$. CTC in this way are able to avoid not only the immune response but also anoikis [18]. To extravasate, CTC use diapedesis to escape the vascular compartment [19]. Then, CTC that present mesenchymal features can inverse their transition and then recover their epithelial phenotype of origin via a process called Mesenchymal to epithelial transition (MET). Some CTC finally become quiescent in a new and favorable environment and can later on fully participate to cancer relapses (Figure 1(e)).

\section{EMT Conditions and Molecular Regulation}

If CTC are the substrate, EMT might be a necessary condition for cancer dissemination. EMT is indeed thought to be the program that cancer cells follow to acquire metastatic features. This substantially simplifies our conception of the metastatic cascade, even if EMT is certainly not sufficient. EMT/MET is a normal embryologic reversible program that allows the conversion of epithelial cells to mesenchymal cells and inversely during development. Its embryonic implication, especially in gastrulation, neural crest delamination, and organ formation and development, is well described [20]. Later, in response to injuries, EMT was shown to be induced by EGF [21] and used by keratinocytes in healing process [22].

The corner stone of EMT/MET processes is the down-/ upregulation of E-Cadherin (E-Cad), an integral membrane protein and a component of adherent junctions and an important mediator of cell-cell adhesion. The $C D H 1$ gene encodes E-Cad. It can be repressed in two ways, depending on the effect on the E-cadherin promoter. First, transcriptional repressors including Snail, Slug, ZEB1, and ZEB2 (zinc finger proteins) and basic helix-loop-helix (bHLH) such as E47 transcription factor bind directly to E-boxes of the $\mathrm{CDH1}$ promoter region [23-26]. Kruppel-like Factor 8 also represses
E-Cad expression by fixing $C D H 1$ promoter in an E-box independent way [27]. Second, the bHLH Twistl factor, E22 factors, and the embryonic transcription factor Goosecoid indirectly repress the $\mathrm{CDH} 1$ transcription $[28,29]$. Interestingly, Snail and Twist appear to control positively ZEB1 expression [30].

Many EMT inducers are currently known. Nuclear factor kappa-B (NF- $\kappa$ B) has a putative binding site on the Snail promoter, inducing Snail protein and preventing its phosphorylation by glycogen synthase kinase-3 (GSK-3) and its subsequent degradation [31]. It has been shown that tumor necrosis factor $\alpha$ (TNF- $\alpha$ ) induces and stabilizes Snail protein via NF- $\kappa \mathrm{B}$ [32]. Transforming growth factor- $\beta$ (TGF- $\beta$ ) is a well-known EMT inducer. The binding of TGF- $\beta$ to its receptor leads to phosphorylation of Smad transcription factors, which strongly induce Snail and Twist expression, particularly in presence of high-mobility group protein HMGA 2 [33]. Protein kinase A (PKA), signal transducer and activator of transcription 3 (STAT3), and protein kinase D (PKD) are involved in TGF- $\beta$-induced EMT $[34,35]$.

Local conditions could also modulate the EMT process. This is the case of hypoxia, a local condition frequently encountered in the tumor mass. Indeed, during hypoxia, Notch pathway is activated, resulting in Notch intracellular domain (NICD) liberation. NICD acts then as a transcription factor that interacts with DNA-binding protein CSL to regulate gene expression. NICD particularly upregulates Snail expression by direct binding to its promoter [36]. Similarly, still in hypoxic condition, hypoxia-inducible factor-1 (HIF-1), potentiated by Notch, is able to stabilize Snail by recruiting lysyl-oxidase (LOX) [36]. However, HIF-1 can also induce Twist expression by binding directly to the hypoxia-response element (HRE) to the Twist promoter sequence [37]. As another factor was upregulated during hypoxia or inflammation, vascular-endothelial growth factor or VEGF can induce Twist and Snail expression by GSK-3 inhibition [38, 39]. The same regulation of Twist and Snail expression is observed with EGF as it can particularly act in cooperation with $\alpha 5 \beta 1$ integrin [40]. Sonic-Hedgehog pathway is also related to Snail expression, probably induced by Gli1 [41] and contributes to TGF- $\beta$-induced EMT [42]. Hyperactive Wnt signaling occurs with the progression of different carcinomas and it has been shown that Wnt stabilizes Snail (and therefore EMT) by GSK3B inhibition via Axin-2 [43]. Thus, EMT appears to be the result of E-Cad repressors activities, especially Snail factors, in response to inflammation and hypoxic conditions [44], both features that are met in cancer.

On the other side, another pathway including miRNAs is well known for its rule in epithelial transition. Bone morphogenetic protein (BMP) pathway, especially via BMP7, induces miR-205 and miR-200 family of microRNAs, which induce $\mathrm{CDH1}$ promoter and suppress ZEB1 and ZEB2 expression [45], and thus promotes MET [46] (Figure 1(e)).

\section{EMT-Related Changes}

During EMT, epithelial cancer cells, which lean bit by bit towards the mesenchymal state, loose epithelial features and 




FIGURE 1: Insights on GBM dissemination process. Both GSC and differentiated cells can undergo EMT in order to invade the brain parenchyma. This process is regulated by different transcription factors including ZEB, SNAIL, Twist, or NF- $\kappa$ B that are activated upon several environmental conditions (inflammation, necrosis, and hypoxia) ((a) and (b)). This consequently results in the acquisition of mesenchymal properties and the expression of ECM degrading enzymes in order to favor tumor spread. This process also sustains intravasation, leading to systemic dissemination ( (c) and (d)). Tumor blood vessels are usually incomplete and leaky, therefore favoring intra-/extravasation (d). In pathological conditions, the BBB is often disrupted, facilitating GBM cells to jump in the blood flow as well (d). When tumor cells extravasate, they may either become quiescent or develop metastases. This balance is tightly regulated by environmental conditions and factors including BMP7 or TGF $\beta 2$ among many others which may induce either dormancy or a switch toward MET and metastases (e). 
change their protein expression. Hence, it is possible to characterize the CTC epithelial or mesenchymal phenotype and specifically the degree of transition. Epithelial cellular adhesion molecule (EpCAM) [47], cytokeratins [48], zonula occludens [49], or epithelial splicing regulator 1 (ESPR1) [50] expression characterize an epithelial phenotype, while $\mathrm{N}$ Cadherin [51] or Vimentin [52] are expressed in mesenchymal phenotype. Activation of biochemical pathways, such as Twist-1 or the Akt-PI3K pathway [53], can also be specific hallmarks of the mesenchymal state. EMT is associated with the acquisition of several properties that are critical for cancer dissemination including first repression of the epithelial cell polarity and proliferation, and second, promotion of cell resistance to therapy, migration, and invasion [20]. Inversely, MET promotes cell proliferation and metastasis formation.

E-Cad repressors as well as EMT inducers are involved in this acquisition. For example, Snail factors induce MMP9 expression that is then able to degrade the basement membrane of blood vessels, a prerequisite step to intravasation. Conversely, some metalloproteases, such as MMP3 and MMP-13, can induce EMT [54, 55]. Additionally, TGF- $\beta$ confers resistance to cell death and DNA damage [56]. In fact, Snail and Slug factors repress proapoptotic genes expression, in particular PUMA, ATM, and PTEN that are usually upregulated in the p53-mediated apoptotic pathway [57]. In the same line, Twistl and Twist 2 were shown to be overexpressed in a large fraction of human cancers and are thus able to override the oncogene-induced premature senescence by abrogating key regulators of the p53- and Rb-dependent pathways. In epithelial cells, the oncogenic cooperation between Twist proteins and activated mitogenic oncoproteins led to complete EMT. Taken together, these data underlined an unexpected link between early escape from failsafe programs and the acquisition of invasive features by cancer cells [58]. EMT is also associated with chemo- and radio-resistance. Snail indeed inactivates p53mediated apoptosis [57], whereas Twist upregulates the serine/threonine kinase AKT2 [59]. Finally, Kudo-Saito et al. showed in melanoma that Snail positive tumor cells have recourse to thrombospondin-1 (TSP-1) in order to impair dendritic cells, resulting in $\mathrm{CD} 4+$ regulatory $\mathrm{T}$ cells induction with immunosuppressive capacity, hence promoting immunoresistance, immunosuppression, and/or escape of immune surveillance [60].

Mesenchymal transition also appears to confer or enhance stem cell properties by activation of Ras/MAPK pathway [61] (Figures 1(a) and 1(b)). Snail factors can indeed promote the Wnt pathway (known for its regulation in selfrenewal and differentiation in stem cells) by E-Cad repression [62]. ZEB1 and ZEB2 factors downregulate some specific members of the microRNAs 200 family (miR-200), particularly miR-200c, which targets the polycomb group member BMI1, an essential regulator of stem-cell renewal, acting as a repressor of various genes by modulating the chromatin status $[45,63,64]$. More and more reports highlight the importance of the miR-200/ZEB feedback loop in determining epithelial and mesenchymal future of tumor cells [64]. In the same way, Lu et al. used the loop to define three different states in the continuum between the epithelial and mesenchymal differentiation: epithelial (high miR-200/low ZEB), mesenchymal (low miR-200/high ZEB), and partial EMT (medium miR-200/medium ZEB) [65]. The acquisition of stem cell properties could explain the possible various origins of CSC.

No matter the epithelial or mesenchymal state, some cell markers suggest stemness character in CTC. In breast cancer for instance, aldehyde dehydrogenase-1 (ALDH1) allows to detect CTC with CSC properties [53]. The expression of cell surface markers CD44+/CD24- is also associated with CSC in breast carcinoma and with CTSC in colon carcinomas [66]. Gangliosides (GD2, GD3, and GD1A) in breast cancer and $\mathrm{ABC}$ proteins (ABCG2) in lung cancer are also useful for stemness detection $[67,68]$, but their utility for CTSC detection remains uncertain.

\section{Dormancy}

Tumor cells that are physically separated from the primary tumor mass and have spread to other anatomical locations through circulation are called disseminated tumor cells (DTC). They can be classified as a subgroup of CTC. Metastasis formation is one option that DTC can follow but some of them are also able to become quiescent, a process that is different from senescence and consists in a nonproliferative state consequent to cell cycle arrest in phase G0/G1 [69]. Quiescence results from mitogenic signaling reduction and implies autophagy [70], reduced PI3K-AKT signaling [71], and activation of stress signaling pathways [72]. Interestingly, dormancy is significantly influenced by the microenvironment which can be permissive or restrictive [73]. In the bone marrow compartment, the presence of proteins such as GAS6, BMP4, BMP7, and TGF- $\beta 2$ confers an adequate environment for dormancy [74-76], whereas VCAM1, periostin, and extracellular matrix stiffness, with high density of type I collagen, appear to induce escape of dormancy [77-79]. Many key players modulate tumor cells dormancy. Among them, the balance of two prominent pathways, p38 mitogen-activated protein kinase (MAPK) and extracellular signal-regulated kinase (ERK), might be key determining factors [75]. A high ratio of ERK/p38 is observed in metastatic lesions [80], while low ratio of ERK/p38 is associated with dormancy [81]. In addition, inactivation of Myc oncogene also leads to senescence [82].

Multiple actors are involved in quiescence process. For example, fibroblasts express periostin, which recruits Wnt pathway ligands and increases Wnt signaling in cancer stem cells, resulting in metastatic colonization [83]. In the bone marrow stem cell niche, stromal cells, such as osteoblasts, via TGF- $\beta 2$, induce low radio of ERK/p-38 and p27 expression, inhibit CDK4, and in this way induce cancer cell quiescence [81]. In the same way, bone morphogenetic protein 7 (BMP-7) binds to BMPR2 that activates p38 and increases the expression of cell cycle inhibitor p21 and metastasis suppressor gene NDRG1 (N-myc downstream-regulated Gene 1) [75]. Macrophages, CD4+ and CD8+ T cells, founded in immune niche, use tumor necrosis factor receptor 1 (TNFR1) and interferon- $\gamma($ IFN- $\gamma$ ) in order to induce antiangiogenic chemokines and prevent proliferation and carcinogenesis 
[84]. More specifically, CD4+ T cells products CXCL9 and CXCL10, which were described, inhibit angiogenesis [85]. Endothelial cells from bone marrow vascular niches can also induce quiescence via TSP-1 or perlecan production $[78,86]$.

Dormancy appears as an important phenomenon in the cancer relapse as it implies higher resistance against targeted and conventional therapies, and after long period, sometimes decades, tumor cells can quit this specific dormancy state and develop regrowth capacities [87]. A strong link between dormancy state and tumor stem cells is suspected [88]. Indeed, both dormant tumor cells and tumor stem cells show a high resistance to current treatments $[89,90]$ and can undergo cell cycle arrest in response to different form of therapy [91, 92]. In glioblastoma, for example, the CSC pool in tumors is enriched after ionizing radiation. This situation seems to be in direct consequence with the activation of DNA damage repair pathways coupled to a reduction of proliferation and apoptosis via DNA checkpoint kinases [93]. In fact, a subpopulation of CSC is thought to be quiescent [94]. This view is supported by the fact that dormant cells and CSC use the same pathways such as Shh, Notch, and Wnt [95]. The overlap between dormancy state and ability of tumor-initiation could help to determinate the subpopulation of tumor cells, which are highly involved in relapses.

\section{CTSC and Glioblastoma}

6.1. Clinical Evidence. GBM is the most frequent primary brain tumor and is well known for its poor prognosis despite multimodal therapies. The rapid relapse of tumor in GBM patients has indeed been regarded for years as the major cause of the lack of GBM spread out of the central nervous system. However, there are several clinical descriptions of glioblastoma metastasis. In 1928, Davis and colleagues described the first case ever reported of glioblastoma metastasis in a 31year-old woman. Since then, a growing body of evidence has shown the capacity of GBM to spread not only via the cerebrospinal fluid (CSF) but also via blood or lymphatic vessels [96, 97] (Figure 1(e)). Interestingly, the number of GBM metastatic reports increases progressively [98]. This could be explained by a higher rate of diagnosis not only due to imaging improvement but also due to the modest but real increase of patient survival and outcomes. Interestingly, the incidence of glioma metastases on postmortem examinations ranges from 6 to $25 \%$ for supratentorial tumors $[99,100]$. The actual delay between the initial tumor diagnosis and metastases found in the literature is 1 to 60 months [101]. Thus, clinical evidences allow to asserting the existence of CTC and DTC in GBM.

6.2. CSC in Glioblastoma. Ignatova et al. first highlighted the presence of CSC in GBM [102] (Figure 1(a)). Many similarities exist between GSC and normal stem cells in the adult brain, also termed neural stem cells (NSC). These populations indeed share particular resemblances in gene expression and signaling pathways including Notch, Wnt, or TGF- $\beta$ signaling [103-105]. CD133 or prominin-1 was proposed as a biomarker of tumor progression/initiation cells described in glioblastoma [106], but it appeared later to be insufficient as CD133-negative cells were also able to initiate tumors [107]. Interestingly, not only Sox 2 (a transcription factor) but also nestin (an intermediate filament protein) and integrin $\alpha 6$ expression are highly expressed in GSC population $[108,109]$. EGFR, whose amplification and mutations are well known in GBM, also promotes stemness in GBM cells [110]. Although it is unclear whether GSC result from cancerous transformation of NSC, they have been demonstrated to preferentially locate in specific niches, more specifically in neurogenic niches, such as subventricular zone [111]. Evidence also considers their presence in necrotic niches [112] or in tumor edge niches [113].

6.3. Defective Brain-Blood Barrier (BBB) and GBM-Circulating Cells. The blood brain barrier (BBB) consists basically of endothelial cells connected by tight junctions, surrounded by astrocytic endfeet with pericytes embedded in the vessel basal membrane. Nevertheless, neurons and microglia are also implicated in the BBB cytoarchitecture [114]. In fact, a double interaction exists between endothelial cells and astrocytes, called gliovascular coupling. While endothelial cell can stimulate astrocytic growth and differentiation, astrocytes also modulate tight junctions formation and angiogenesis via the src-suppressed C-kinase substrate (SSeCKS) [115, 116]. Moreover, astrocytic endfeet use aquaporins (AQP) to maintain the BBB integrity [117].

GBM is the most vascularized tumor in humans [118]. Among others, this can be explained by high levels of vascular endothelial growth factor (VEGF), particularly in necrotic core, resulting in endothelial proliferation [119]. Nevertheless, glioblastoma-induced angiogenesis is imperfect leading to vessel formation with variable diameter and permeability, heterogeneous distribution, and basal lamina irregularities [120] (Figures 1(c) and 1(d)). In 1975, Hirano and Matsui had already shown fenestrations and tight junctions disruption in GBM vessels [121]. At the beginning, GBM cells use host vessels as pathways of invasion [122] and, then, co-opt to these vessels [123]. These interactions of GBM cells with vessels become more and more prominent as the disease progresses. Indeed, new-generated vessels by angiogenesis can support tumor growth, with a tone controlled by glioma cells [124]. Watkins et al. also showed that glioma cells displace, or even eliminate, astrocytic endfeet and make direct contacts with endothelial cells (Figures 1(c) and 1(d)). The result is, first, the cessation of endothelial/astrocytic interaction and, second, the breach of BBB, by reduction of tight junctions [124]. Thus, glioblastoma progression seems to tightly associate with altered BBB permeability, which also constitutes the first condition to intravasate.

6.4. Glioblastoma Subtypes and EMT: The Mesenchymal Link. Based on gene expression signatures, four GBM subtypes have been described: proneural, neural, classical, and mesenchymal [125]. In particular, the mesenchymal subtype is characterized by high expression of CHI3L1 and MET, wildtype IDH1, mutation/deletion of NF1, Schwann-like features, and important presence of necrosis/inflammation [125-127]. 
This subtype is usually associated with worse prognosis and most of the time, appears de novo [128, 129]. Fibronectin and collagen $5 \alpha 1$ are used as markers of mesenchymal GBM subtype [125]. Some regulators of mesenchymal status have also been highlighted in this subtype, such as C/EBP-B and STAT3 transcription factors or the transcriptional coactivator TAZ $[130,131]$.

In this context, Bhat and colleagues have recently shown that microglia are able to induce the mesenchymal status via the TNF- $\alpha / \mathrm{NF}-\kappa \mathrm{B}$ pathway, notably resulting in radioresistance [132]. Moreover, it has been shown that the mesenchymal phenotype is associated with higher migratory capacities of GBM cells. In fact, TGF- $\beta$, which is well present in the GBM environment and secreted by microglia, stromal and tumor cells [133], is able to induce the mesenchymal transition, via SMAD2 phosphorylation and recruitment of ZEB1, especially in GBM with a low or absent expression of mesenchymal markers [134]. This mesenchymal differentiation can be effectively blocked by A8301, an inhibitor of the TGF- $\beta$ type 1 receptors [134]. Hypoxia, via HIF- $1 \alpha$ and ZEB1, is also able to induce a mesenchymal switch in GBM [135]. Moreover, Twist overexpression enhances GBM invasion [136]. Snail is also upregulated in glioma cells compared to normal brain cells and was shown to promote invasion [137]. Indeed, its inactivation inhibits glioma progression and migration [138]. Finally, stromal cell-derived factor (SDF-1) or CXCL12 and its receptor CXCR4 can induce EMT in GBM via activation PI3K/Akt and ERK pathways [139]. Interestingly another recent study reported the involvement of the CXCL12/CXCR4 axis in EMT transition via upregulation of survivin, a protein involved in apoptosis inhibition [140]. Moreover survivin-mediated EMT was shown to promote resistance to $\gamma$-radiation, suggesting a potential role of EMT in GBM therapeutic resistance [141].

As mesenchymal transition is associated with the acquisition of stem cell properties, hypoxia seems to increase stemcell markers in GBM cells, via HIF- $1 \alpha$ and Notch inductions [142]. Speaking about new properties, mesenchymal transition in GBM was shown to confer tumor resistance to antiVEGF therapy [143].

GBM metastases are not stricto sensu associated with the mesenchymal subtype. This is no surprise as it has been demonstrated that different subtypes of GBM can coexist within the same tumor [144]. Moreover, Ozawa et al. showed that GBM could derive from a common proneural-like precursor and that additional NF1 loss can convert this proneural subtype to a mesenchymal subtype [145]. Thus, mesenchymal transition can be understood as a late phenomenon in GBM, leading to more aggressive, invasive, and recurrent tumor. This idea is supported by the fact that mesenchymal subtype is frequently found in glioblastoma metastases and recurrences [146].

6.5. CTSC in Glioblastoma. Recently, CTC have been found in GBM patients' blood, highlighted by GFAP detection, EGFR amplification, or increased telomerase activity [147, 148]. The phenotypes of these CTC in GBM patients were closed to the mesenchymal or proneural subtypes. However, recent studies have not found stemness features in those cells yet, but it does not rule out that some of these CTC are also indeed true CTSC. Of course, this hypothesis is sustained by clinical evidences and the existence of GBM metastases [98]. Recently, Song et al. showed that MMP9 is required to cross the $\mathrm{BBB}$, especially the parenchymal barrier [149]. Interestingly, as Snail also induces MMP-9 expression, the mesenchymal transition therefore seems a necessary condition to intravasate (Figure 1(c)). Besides, as dormancy is also a reality in GBM [150], we can speculate that some of these CTC remain quiescent in other tissue and could later on initiate relapses. Moreover, circulating endothelial cells and circulating hematopoietic progenitor cells also appear to be present in GBM [151]. Interestingly, GSC have the ability to differentiate into endothelial cells and show the ability to generate new tumors when grafted in immunodeficient mice [152] (Figure 1(d)). This reinforces even more the hypothesis according to which CTSC are an underrated reality in GBM.

\section{Conclusion}

For many years, GBM was thought to be restricted to the central nervous system but a growing body of evidence indicates that, like many other cancers, hematogenic dissemination is a reality. CTC characterization is needed to confirm the presence of CTSC. The question of a possible CTC role in GBM relapses remains open. We think this is a crucial question to address as its response could significantly modify actual therapeutic protocols and have an important impact on patient outcomes.

\section{Conflict of Interests}

The authors declare they have no conflict of interests.

\section{Acknowledgments}

This work was supported by grants from the National Fund for Scientific Research (F.N.R.S/F.R.I.A), the Special Funds of the University of Liege, and the Anti-Cancer Center near the University of Liège and the Leon Fredericq Grant.

\section{References}

[1] S. Sun and X. S. Qiu, "Cancer stem cells and tumor metastasis," Journal of Cancer Research and Therapeutics, vol. 9, supplement, pp. S150-S152, 2013.

[2] M. F. Clarke, J. E. Dick, P. B. Dirks et al., "Cancer stem cellsperspectives on current status and future directions: AACR workshop on cancer stem cells," Cancer Research, vol. 66, no. 19, pp. 9339-9344, 2006.

[3] D. Hanahan and R. A. Weinberg, "The hallmarks of cancer," Cell, vol. 100, no. 1, pp. 57-70, 2000.

[4] S. K. Singh, I. D. Clarke, M. Terasaki et al., "Identification of a cancer stem cell in human brain tumors," Cancer Research, vol. 63, no. 18, pp. 5821-5828, 2003. 
[5] L. Ricci-Vitiani, D. G. Lombardi, E. Pilozzi et al., "Identification and expansion of human colon-cancer-initiating cells," Nature, vol. 445, no. 7123, pp. 111-115, 2007.

[6] C. E. Eyler and J. N. Rich, "Survival of the fittest: cancer stem cells in therapeutic resistance and angiogenesis," Journal of Clinical Oncology, vol. 26, no. 17, pp. 2839-2845, 2008.

[7] W. A. Woodward, M. S. Chen, F. Behbod, M. P. Alfaro, T. A. Buchholz, and J. M. Rosen, "WNT/beta-catenin mediates radiation resistance of mouse mammary progenitor cells," Proceedings of the National Academy of Sciences of the United States of America, vol. 104, no. 2, pp. 618-623, 2007.

[8] G. Rahmathulla, S. A. Toms, and R. J. Weil, "The molecular biology of brain metastasis," Journal of Oncology, vol. 2012, Article ID 723541, 16 pages, 2012.

[9] C. Scheel and R. A. Weinberg, "Cancer stem cells and epithelialmesenchymal transition: concepts and molecular links," Seminars in Cancer Biology, vol. 22, no. 5-6, pp. 396-403, 2012.

[10] T. Reya, S. J. Morrison, M. F. Clarke, and I. L. Weissman, "Stem cells, cancer, and cancer stem cells," Nature, vol. 414, no. 6859, pp. 105-111, 2001.

[11] S. Liu, Y. Cong, D. Wang et al., "Breast cancer stem cells transition between epithelial and mesenchymal states reflective of their normal counterparts," Stem Cell Reports, vol. 2, no. 1, pp. 78-91, 2014.

[12] N. Moro, C. Mauch, and P. Zigrino, "Metalloproteinases in melanoma," European Journal of Cell Biology, vol. 93, no. 1-2, pp. 23-29, 2014.

[13] C. Lewis and C. Murdoch, "Macrophage responses to hypoxia: implications for tumor progression and anti-cancer therapies," American Journal of Pathology, vol. 167, no. 3, pp. 627-635, 2005.

[14] D. F. Quail and J. A. Joyce, "Microenvironmental regulation of tumor progression and metastasis," Nature Medicine, vol. 19, no. 11, pp. 1423-1437, 2013.

[15] B. Nieswandt, M. Hafner, B. Echtenacher, and D. N. Männel, "Lysis of tumor cells by natural killer cells in mice is impeded by platelets," Cancer Research, vol. 59, no. 6, pp. 1295-1300, 1999.

[16] P. S. Steeg, "Tumor metastasis: mechanistic insights and clinical challenges," Nature Medicine, vol. 12, no. 8, pp. 895-904, 2006.

[17] L. J. Gay and B. Felding-Habermann, "Contribution of platelets to tumour metastasis," Nature Reviews Cancer, vol. 11, no. 2, pp. 123-134, 2011.

[18] J. Grossmann, "Molecular mechanisms of 'detachment-induced apoptosis-Anoikis," Apoptosis, vol. 7, no. 3, pp. 247-260, 2002.

[19] Y. Kienast, L. Von Baumgarten, M. Fuhrmann et al., "Real-time imaging reveals the single steps of brain metastasis formation," Nature Medicine, vol. 16, no. 1, pp. 116-122, 2010.

[20] J. P. Thiery, H. Acloque, R. Y. J. Huang, and M. A. Nieto, "Epithelial-mesenchymal transitions in development and disease," Cell, vol. 139, no. 5, pp. 871-890, 2009.

[21] N. Ahmed, S. Maines-Bandiera, M. A. Quinn, W. G. Unger, S. Dedhar, and N. Auersperg, "Molecular pathways regulating EGF-induced epithelio-mesenchymal transition in human ovarian surface epithelium," The American Journal of Physiology-Cell Physiology, vol. 290, no. 6, pp. C1532-C1542, 2006.

[22] V. Arnoux, M. Nassour, A. L'Helgoualc'h, R. A. Hipskind, and P. Savagner, "Erk5 controls slug expression and keratinocyte activation during wound healing," Molecular Biology of the Cell, vol. 19, no. 11, pp. 4738-4749, 2008.

[23] E. Batlle, E. Sancho, C. Franci et al., "The transcription factor Snail is a repressor of E-cadherin gene expression in epithelial tumour cells," Nature Cell Biology, vol. 2, no. 2, pp. 84-89, 2000.
[24] A. Eger, K. Aigner, S. Sonderegger et al., "DeltaEF1 is a transcriptional repressor of E-cadherin and regulates epithelial plasticity in breast cancer cells," Oncogene, vol. 24, no. 14, pp. 2375-2385, 2005.

[25] J. Comijn, G. Berx, P. Vermassen et al., “The two-handed E box binding zinc finger protein SIP1 downregulates E-cadherin and induces invasion," Molecular Cell, vol. 7, no. 6, pp. 1267-1278, 2001.

[26] M. A. Pérez-Moreno, A. Locascio, I. Rodrigo et al., "A new role for E12/E47 in the repression of E-cadherin expression and epithelial-mesenchymal transitions," Journal of Biological Chemistry, vol. 276, no. 29, pp. 27424-27431, 2001.

[27] X. Wang, M. Zheng, G. Liu et al., "Krüppel-like factor 8 induces epithelial to mesenchymal transition and epithelial cell invasion," Cancer Research, vol. 67, no. 15, pp. 7184-7193, 2007.

[28] J. Yang and R. A. Weinberg, "Epithelial-mesenchymal transition: at the crossroads of development and tumor metastasis," Developmental Cell, vol. 14, no. 6, pp. 818-829, 2008.

[29] V. R. Sobrado, G. Moreno-Bueno, E. Cubillo et al., "The class I bHLH factors E2-2A and E2-2B regulate EMT," Journal of Cell Science, vol. 122, no. 7, pp. 1014-1024, 2009.

[30] N. Dave, S. Guaita-Esteruelas, S. Gutarra et al., "Functional cooperation between snaill and twist in the regulation of ZEB1 expression during epithelial to mesenchymal transition," The Journal of Biological Chemistry, vol. 286, no. 14, pp. 12024-12032, 2011.

[31] Y. Wu, J. Deng, P. G. Rychahou, S. Qiu, B. M. Evers, and B. P. Zhou, "Stabilization of snail by NF-kappaB is required for inflammation-induced cell migration and invasion," Cancer Cell, vol. 15, no. 5, pp. 416-428, 2009.

[32] R. Dong, Q. Wang, X. L. He, Y. K. Chu, J. G. Lu, and Q. J. Ma, "Role of nuclear factor kappa $\mathrm{B}$ and reactive oxygen species in the tumor necrosis factor- $\alpha$-induced epithelial-mesenchymal transition of MCF-7 cells," Brazilian Journal of Medical and Biological Research, vol. 40, no. 8, pp. 1071-1078, 2007.

[33] S. Thuault, E.-J. Tan, H. Peinado, A. Cano, C.-H. Heldin, and A. Moustakas, "HMGA2 and Smads co-regulate SNAIL1 expression during induction of epithelial-to-mesenchymal transition," The Journal of Biological Chemistry, vol. 283, no. 48, pp. 3343733446, 2008.

[34] Y. Yang, X. Pan, W. Lei, J. Wang, and J. Song, “Transforming growth factor- $\beta 1$ induces epithelial-to-mesenchymal transition and apoptosis via a cell cycle-dependent mechanism," Oncogene, vol. 25, no. 55, pp. 7235-7244, 2006.

[35] Y. Yang, X. Pan, W. Lei et al., "Regulation of transforming growth factor- $\beta 1$-induced apoptosis and epithelial-tomesenchymal transition by protein kinase A and signal transducers and activators of transcription 3," Cancer Research, vol. 66, no. 17, pp. 8617-8624, 2006.

[36] C. Sahlgren, M. V. Gustafsson, S. Jin, L. Poellinger, and U. Lendahl, "Notch signaling mediates hypoxia-induced tumor cell migration and invasion," Proceedings of the National Academy of Sciences of the United States of America, vol. 105, no. 17, pp. 6392-6397, 2008.

[37] M. H. Yang, M. Z. Wu, S. H. Chiou et al., "Direct regulation of TWIST by HIF-1alpha promotes metastasis," Nature Cell Biology, vol. 10, no. 3, pp. 295-305, 2008.

[38] A. D. Yang, E. R. Camp, F. Fan et al., "Vascular endothelial growth factor receptor-1 activation mediates epithelial to mesenchymal transition in human pancreatic carcinoma cells," Cancer Research, vol. 66, no. 1, pp. 46-51, 2006. 
[39] L. S. Wanami, H.-Y. Chen, S. Peiró, A. García de Herreros, and R. E. Bachelder, "Vascular endothelial growth factor-A stimulates Snail expression in breast tumor cells: implications for tumor progression," Experimental Cell Research, vol. 314, no. 13, pp. 2448-2453, 2008.

[40] M.-Y. Lee, C.-Y. Chou, M.-J. Tang, and M.-R. Shen, "Epithelialmesenchymal transition in cervical cancer: correlation with tumor progression, epidermal growth factor receptor overexpression, and snail up-regulation," Clinical Cancer Research, vol. 14 , no. 15 , pp. 4743-4750, 2008.

[41] V. Fendrich, J. Waldmann, F. Esni et al., "Snail and Sonic Hedgehog activation in neuroendocrine tumors of the ileum," Endocrine-Related Cancer, vol. 14, no. 3, pp. 865-874, 2007.

[42] M. Y. Maitah, S. Ali, A. Ahmad, S. Gadgeel, and F. H. Sarkar, "Up-regulation of sonic hedgehog contributes to TGF- $\beta 1$ induced epithelial to mesenchymal transition in NSCLC cells," PLoS ONE, vol. 6, no. 1, Article ID e16068, 2011.

[43] J. I. Yook, X.-Y. Li, I. Ota et al., "A Wnt-Axin2-GSK3 $\beta$ cascade regulates Snaill activity in breast cancer cells," Nature Cell Biology, vol. 8, no. 12, pp. 1398-1406, 2006.

[44] J. M. López-Nouoa and M. A. Nieto, "Inflammation and EMT: an alliance towards organ fibrosis and cancer progression," EMBO Molecular Medicine, vol. 1, no. 6-7, pp. 303-314, 2009.

[45] M. Korpal, E. S. Lee, G. Hu, and Y. Kang, "The miR-200 family inhibits epithelial-mesenchymal transition and cancer cell migration by direct targeting of E-cadherin transcriptional repressors ZEB1 and ZEB2," Journal of Biological Chemistry, vol. 283, no. 22, pp. 14910-14914, 2008.

[46] P. Samavarchi-Tehrani, A. Golipour, L. David et al., "Functional genomics reveals a BMP-driven mesenchymal-to-epithelial transition in the initiation of somatic cell reprogramming," Cell Stem Cell, vol. 7, no. 1, pp. 64-77, 2010.

[47] C. G. Rao, D. Chianese, G. V. Doyle et al., "Expression of epithelial cell adhesion molecule in carcinoma cells present in blood and primary and metastatic tumors," International Journal of Oncology, vol. 27, no. 1, pp. 49-57, 2005.

[48] J. P. Thiery and J. P. Sleeman, "Complex networks orchestrate epithelial-mesenchymal transitions," Nature Reviews Molecular Cell Biology, vol. 7, no. 2, pp. 131-142, 2006.

[49] E. A. Runkle and D. Mu, "Tight junction proteins: from barrier to tumorigenesis," Cancer Letters, vol. 337, no. 1, pp. 41-48, 2013.

[50] C. C. Warzecha, S. Shen, Y. Xing, and R. P. Carstens, "The epithelial splicing factors ESRP1 and ESRP2 positively and negatively regulate diverse types of alternative splicing events," RNA biology, vol. 6, no. 5, pp. 546-562, 2009.

[51] R. B. Hazan, R. Qiao, R. Keren, I. Badano, and K. Suyama, "Cadherin switch in tumor progression," Annals of the New York Academy of Sciences, vol. 1014, pp. 155-163, 2004.

[52] M. B. Lustberg, P. Balasubramanian, B. Miller et al., "Heterogeneous atypical cell populations are present in blood of metastatic breast cancer patients," Breast Cancer Research, vol. 16, no. 2, article R23, 2014.

[53] G. Barriere, A. Riouallon, J. Renaudie, M. Tartary, and M. Rigaud, "Mesenchymal characterization: alternative to simple CTC detection in two clinical trials," Anticancer Research, vol. 32, no. 8, pp. 3363-3370, 2012.

[54] D. C. Radisky, D. D. Levy, L. E. Littlepage et al., "Raclb and reactive oxygen species mediate MMP-3-induced EMT and genomic instability," Nature, vol. 436, no. 7047, pp. 123-127, 2005.
[55] C. Billottet, M. Tuefferd, D. Gentien et al., "Modulation of several waves of gene expression during FGF-1 induced epithelialmesenchymal transition of carcinoma cells," Journal of Cellular Biochemistry, vol. 104, no. 3, pp. 826-839, 2008.

[56] S. Vega, A. V. Morales, O. H. Ocaña, F. Valdés, I. Fabregat, and M. A. Nieto, "Snail blocks the cell cycle and confers resistance to cell death," Genes and Development, vol. 18, no. 10, pp. 1131-1143, 2004.

[57] N. K. Kurrey, S. P. Jalgaonkar, A. V. Joglekar et al., "Snail and slug mediate radioresistance and chemoresistance by antagonizing p53-mediated apoptosis and acquiring a stem-like phenotype in ovarian cancer cells," Stem Cells, vol. 27, no. 9, pp. 2059-2068, 2009.

[58] S. Ansieau, J. Bastid, A. Doreau et al., "Induction of EMT by twist proteins as a collateral effect of tumor-promoting inactivation of premature senescence," Cancer Cell, vol. 14, no. 1, pp. 79-89, 2008.

[59] G. Z. Cheng, J. Chan, Q. Wang, W. Zhang, C. D. Sun, and L.-H. Wang, "Twist transcriptionally up-regulates AKT2 in breast cancer cells leading to increased migration, invasion, and resistance to paclitaxel," Cancer Research, vol. 67, no. 5, pp. 19791987, 2007.

[60] C. Kudo-Saito, H. Shirako, T. Takeuchi, and Y. Kawakami, "Cancer metastasis is accelerated through immunosuppression during Snail-induced EMT of cancer cells," Cancer Cell, vol. 15, no. 3, pp. 195-206, 2009.

[61] S. A. Mani, W. Guo, M.-J. Liao et al., "The epithelial-mesenchymal transition generates cells with properties of stem cells," Cell, vol. 133, no. 4, pp. 704-715, 2008.

[62] V. Stemmer, B. de Craene, G. Berx, and J. Behrens, "Snail promotes Wnt target gene expression and interacts with $\beta$ catenin," Oncogene, vol. 27, no. 37, pp. 5075-5080, 2008.

[63] U. Wellner, J. Schubert, U. C. Burk et al., “The EMT-activator ZEB1 promotes tumorigenicity by repressing stemnessinhibiting microRNAs," Nature Cell Biology, vol. 11, no. 12, pp. 1487-1495, 2009.

[64] L. Hill, G. Browne, and E. Tulchinsky, "ZEB/miR-200 feedback loop: at the crossroads of signal transduction in cancer," International Journal of Cancer, vol. 132, no. 4, pp. 745-754, 2012.

[65] M. Lu, M. K. Jolly, H. Levine, J. N. Onuchic, and E. Ben-Jacob, "MicroRNA-based regulation of epithelial-hybridmesenchymal fate determination," Proceedings of the National Academy of Sciences of the United States of America, vol. 110, no. 45, pp. 18144-18149, 2013.

[66] M. Todaro, M. Gaggianesi, V. Catalano et al., "CD44v6 is a marker of constitutive and reprogrammed cancer stem cells driving colon cancer metastasis," Cell Stem Cell, vol. 14, no. 3, pp. 342-356, 2014.

[67] V. L. Battula, Y. Shi, K. W. Evans et al., "Ganglioside GD2 identifies breast cancer stem cells and promotes tumorigenesis," The Journal of Clinical Investigation, vol. 122, no. 6, pp. 20662078, 2012.

[68] M. M. Ho, A. V. Ng, S. Lam, and J. Y. Hung, "Side population in human lung cancer cell lines and tumors is enriched with stemlike cancer cells," Cancer Research, vol. 67, no. 10, pp. 4827-4833, 2007.

[69] M. S. Sosa, P. Bragado, and J. A. Aguirre-Ghiso, "Mechanisms of disseminated cancer cell dormancy: an awakening field," Nature Reviews Cancer, vol. 14, no. 9, pp. 611-622, 2014.

[70] D. M. Schewe and J. A. Aguirre-Ghiso, "ATF6 $\alpha$-Rheb-mTOR signaling promotes survival of dormant tumor cells in vivo," 
Proceedings of the National Academy of Sciences of the United States of America, vol. 105, no. 30, pp. 10519-10524, 2008.

[71] H. Jo, Y. Jia, K. K. Subramanian, H. Hattori, and H. R. Luo, "Cancer cell-derived clusterin modulates the phosphatidylinositol 3 '-kinase-Akt pathway through attenuation of insulinlike growth factor 1 during serum deprivation," Molecular and Cellular Biology, vol. 28, no. 13, pp. 4285-4299, 2008.

[72] M. S. Sosa, A. Avivar-Valderas, P. Bragado, H.-C. Wen, and J. A. Aguirre-Ghiso, "ERK1/2 and p38alpha/beta signaling in tumor cell quiescence: opportunities to control dormant residual disease," Clinical Cancer Research, vol. 17, no. 18, pp. 5850-5857, 2011.

[73] P. Bragado, M. S. Sosa, P. Keely, J. Condeelis, and J. A. AguirreGhiso, "Microenvironments dictating tumor cell dormancy," Recent Results in Cancer Research, vol. 195, pp. 25-39, 2012.

[74] Y. Shiozawa, A. M. Havens, K. J. Pienta, and R. S. Taichman, "The bone marrow niche: habitat to hematopoietic and mesenchymal stem cells, and unwitting host to molecular parasites," Leukemia, vol. 22, no. 5, pp. 941-950, 2008.

[75] A. Kobayashi, H. Okuda, F. Xing et al., "Bone morphogenetic protein 7 in dormancy and metastasis of prostate cancer stemlike cells in bone," Journal of Experimental Medicine, vol. 208, no. 13, pp. 2641-2655, 2011.

[76] H. Gao, G. Chakraborty, A. P. Lee-Lim et al., "The BMP inhibitor Coco reactivates breast cancer cells at lung metastatic sites," Cell, vol. 150, no. 4, pp. 764-779, 2012.

[77] G. Cheng, J. Tse, R. K. Jain, L. L. Munn, and M. V. Blagosklonny, "Micro-environmental mechanical stress controls tumor spheroid size and morphology by suppressing proliferation and inducing apoptosis in cancer cells," PLOS ONE, vol. 4, no. 2, Article ID e4632, 2009.

[78] C. M. Ghajar, H. Peinado, H. Mori et al., "The perivascular niche regulates breast tumour dormancy," Nature Cell Biology, vol. 15, no. 7, pp. 807-817, 2013.

[79] X. Lu, E. Mu, Y. Wei et al., "VCAM-1 promotes osteolytic expansion of indolent bone micrometastasis of breast cancer by engaging $\alpha 4 \beta 1$-positive osteoclast progenitors," Cancer Cell, vol. 20, no. 6, pp. 701-714, 2011.

[80] J. A. Aguirre-Ghiso, L. Ossowski, and S. K. Rosenbaum, "Green fluorescent protein tagging of extracellular signal-regulated kinase and p38 pathways reveals novel dynamics of pathway activation during primary and metastatic growth," Cancer Research, vol. 64, no. 20, pp. 7336-7345, 2004.

[81] P. Bragado, Y. Estrada, F. Parikh et al., "TGF- $\beta 2$ dictates disseminated tumour cell fate in target organs through TGF- $\beta$ RIII and p38 $\alpha / \beta$ signalling," Nature Cell Biology, vol. 15, no. 11, pp. 1351-1361, 2013.

[82] C.-H. Wu, J. Van Riggelen, A. Yetil, A. C. Fan, P. Bachireddy, and D. W. Felsher, "Cellular senescence is an important mechanism of tumor regression upon c-Myc inactivation," Proceedings of the National Academy of Sciences of the United States of America, vol. 104, no. 32, pp. 13028-13033, 2007.

[83] I. Malanchi, A. Santamaria-Martínez, E. Susanto et al., "Interactions between cancer stem cells and their niche govern metastatic colonization," Nature, vol. 481, no. 7379, pp. 85-91, 2012.

[84] N. Müller-Hermelink, H. Braumüller, B. Pichler et al., "TNFR1 signaling and IFN-gamma signaling determine whether T cells induce tumor dormancy or promote multistage carcinogenesis," Cancer Cell, vol. 13, no. 6, pp. 507-518, 2008.

[85] A. D. Pardee, D. McCurry, S. Alber, P. Hu, A. L. Epstein, and W. J. Storkus, "A therapeutic OX40 agonist dynamically alters dendritic, endothelial, and $\mathrm{T}$ cell subsets within the established tumor microenvironment," Cancer Research, vol. 70, no. 22, pp. 9041-9052, 2010.

[86] J. W. Franses, A. B. Baker, V. C. Chitalia, and E. R. Edelman, "Stromal endothelial cells directly influence cancer progression," Science Translational Medicine, vol. 3, no. 66, Article ID 66ra5, 2011.

[87] C. A. Klein, "Framework models of tumor dormancy from patient-derived observations," Current Opinion in Genetics and Development, vol. 21, no. 1, pp. 42-49, 2011.

[88] S. Kleffel and T. Schatton, “Tumor dormancy and cancer stem cells: two sides of the same coin?" Advances in Experimental Medicine and Biology, vol. 734, pp. 145-179, 2013.

[89] R. Demicheli, R. Miceli, A. Moliterni et al., "Breast cancer recurrence dynamics following adjuvant $\mathrm{CMF}$ is consistent with tumor dormancy and mastectomy-driven acceleration of the metastatic process," Annals of Oncology, vol. 16, no. 9, pp. 14491457, 2005.

[90] X. Li, M. T. Lewis, J. Huang et al., "Intrinsic resistance of tumorigenic breast cancer cells to chemotherapy," Journal of the National Cancer Institute, vol. 100, no. 9, pp. 672-679, 2008.

[91] P. E. Goss and A. F. Chambers, "Does tumour dormancy offer a therapeutic target?" Nature Reviews Cancer, vol. 10, no. 12, pp. 871-877, 2010.

[92] T. M. Phillips, W. H. McBride, and F. Pajonk, "The response of CD $24^{-/ l o w} / \mathrm{CD} 44^{+}$breast cancer-initiating cells to radiation," Journal of the National Cancer Institute, vol. 98, no. 24, pp. 17771785, 2006.

[93] S. Bao, Q. Wu, R. E. McLendon et al., "Glioma stem cells promote radioresistance by preferential activation of the DNA damage response," Nature, vol. 444, no. 7120, pp. 756-760, 2006.

[94] A. P. Kusumbe and S. A. Bapat, "Cancer stem cells and aneuploid populations within developing tumors are the major determinants of tumor dormancy," Cancer Research, vol. 69, no. 24, pp. 9245-9253, 2009.

[95] N. Takebe, P. J. Harris, R. Q. Warren, and S. P. Ivy, “Targeting cancer stem cells by inhibiting Wnt, Notch, and Hedgehog pathways," Nature Reviews Clinical Oncology, vol. 8, no. 2, pp. 97-106, 2011.

[96] C. D. Lawton, D. T. Nagasawa, I. Yang, R. G. Fessler, and Z. A. Smith, "Leptomeningeal spinal metastases from glioblastoma multiforme: treatment and management of an uncommon manifestation of disease: a review," Journal of Neurosurgery: Spine, vol. 17, no. 5, pp. 438-448, 2012.

[97] G. Kalokhe, S. A. Grimm, J. P. Chandler, I. Helenowski, A. Rademaker, and J. J. Raizer, "Metastatic glioblastoma: case presentations and a review of the literature," Journal of NeuroOncology, vol. 107, no. 1, pp. 21-27, 2012.

[98] M. Shahideh, A. Fallah, D. G. Munoz, and R. Loch MacDonald, "Systematic review of primary intracranial glioblastoma multiforme with symptomatic spinal metastases, with two illustrative patients," Journal of Clinical Neuroscience, vol. 19, no. 8, pp. 1080-1086, 2012

[99] K. Onda, R. Tanaka, H. Takahashi, N. Takeda, and F. Ikuta, "Cerebral glioblastoma with cerebrospinal fluid dissemination: a clinicopathological study of 14 cases examined by complete autopsy," Neurosurgery, vol. 25, no. 4, pp. 533-540, 1989.

[100] F. T. Vertosick Jr. and R. G. Selker, "Brain stem and spinal metastases of supratentorial glioblastoma multiforme: a clinical series," Neurosurgery, vol. 27, no. 4, pp. 516-522, 1990. 
[101] W. H. Ng, T. T. Yeo, and A. H. Kaye, "Spinal and extracranial metastatic dissemination of malignant glioma," Journal of Clinical Neuroscience, vol. 12, no. 4, pp. 379-382, 2005.

[102] T. N. Ignatova, V. G. Kukekov, E. D. Laywell, O. N. Suslov, F. D. Vrionis, and D. A. Steindler, "Human cortical glial tumors contain neural stem-like cells expressing astroglial and neuronal markers in vitro," Glia, vol. 39, no. 3, pp. 193-206, 2002.

[103] E. L. Jackson and A. Alvarez-Buylla, "Characterization of adult neural stem cells and their relation to brain tumors," Cells Tissues Organs, vol. 188, no. 1-2, pp. 212-224, 2008.

[104] J. Godlewski, M. O. Nowicki, A. Bronisz et al., "Targeting of the Bmi-1 oncogene/stem cell renewal factor by microRNA-128 inhibits glioma proliferation and self-renewal," Cancer Research, vol. 68, no. 22, pp. 9125-9130, 2008.

[105] Y. Zhu, F. Guignard, D. Zhao et al., "Early inactivation of p53 tumor suppressor gene cooperating with NF1 loss induces malignant astrocytoma," Cancer Cell, vol. 8, no. 2, pp. 119-130, 2005.

[106] S. K. Singh, C. Hawkins, I. D. Clarke et al., "Identification of human brain tumour initiating cells," Nature, vol. 432, no. 7015, pp. 396-401, 2004.

[107] X. Yan, L. Ma, D. Yi et al., "A CD133-related gene expression signature identifies an aggressive glioblastoma subtype with excessive mutations," Proceedings of the National Academy of Sciences of the United States of America, vol. 108, no. 4, pp. 15911596, 2011.

[108] R. M. R. Gangemi, F. Griffero, D. Marubbi et al., "SOX2 silencing in glioblastoma tumor-initiating cells causes stop of proliferation and loss of tumorigenicity," Stem Cells, vol. 27, no. 1, pp. 40-48, 2009.

[109] J. D. Lathia, J. Gallagher, J. M. Heddleston et al., "Integrin alpha 6 regulates glioblastoma stem cells," Cell Stem Cell, vol. 6, no. 5, pp. 421-432, 2010.

[110] X. Jin, J. Yin, S.-H. Kim et al., "EGFR-AKT-Smad signaling promotes formation of glioma stem-like cells and tumor angiogenesis by ID3-driven cytokine induction," Cancer Research, vol. 71, no. 22, pp. 7125-7134, 2011.

[111] F. T. Merkle, A. D. Tramontin, J. M. García-Verdugo, and A. Alvarez-Buylla, "Radial glia give rise to adult neural stem cells in the subventricular zone," Proceedings of the National Academy of Sciences of the United States of America, vol. 101, no. 50, pp. 17528-17532, 2004.

[112] A. M. McCord, M. Jamal, U. T. Shankavarum, F. F. Lang, K. Camphausen, and P. J. Tofilon, "Physiologic oxygen concentration enhances the stem-like properties of $\mathrm{CD}_{133^{+}}$human glioblastoma cells in vitro," Molecular Cancer Research, vol. 7, no. 4, pp. 489-497, 2009.

[113] X.-Z. Ye, S.-L. Xu, Y.-H. Xin et al., “Tumor-associated microglia/macrophages enhance the invasion of glioma stemlike cells via TGF- $\beta 1$ signaling pathway," Journal of Immunology, vol. 189, no. 1, pp. 444-453, 2012.

[114] N. J. Abbott, A. A. K. Patabendige, D. E. M. Dolman, S. R. Yusof, and D. J. Begley, "Structure and function of the blood-brain barrier," Neurobiology of Disease, vol. 37, no. 1, pp. 13-25, 2010.

[115] S.-W. Lee, W. J. Kim, Y. K. Choi et al., "SSeCKS regulates angiogenesis and tight junction formation in blood-brain barrier," Nature Medicine, vol. 9, no. 7, pp. 900-906, 2003.

[116] N. J. Abbott, L. Rönnbäck, and E. Hansson, "Astrocyteendothelial interactions at the blood-brain barrier," Nature Reviews Neuroscience, vol. 7, no. 1, pp. 41-53, 2006.
[117] L. G. Dubois, L. Campanati, C. Righy et al., "Gliomas and the vascular fragility of the blood brain barrier," Frontiers in Cellular Neuroscience, vol. 8, article 418, 2014.

[118] S. Takano, T. Yamashita, and O. Ohneda, "Molecular therapeutic targets for glioma angiogenesis," Journal of Oncology, vol. 2010, Article ID 351908, 11 pages, 2010.

[119] G. Bergers and L. E. Benjamin, "Tumorigenesis and the angiogenic switch," Nature Reviews Cancer, vol. 3, no. 6, pp. 401-410, 2003.

[120] R. K. Jain, E. di Tomaso, D. G. Duda, J. S. Loeffler, A. G. Sorensen, and T. T. Batchelor, "Angiogenesis in brain tumours," Nature Reviews Neuroscience, vol. 8, no. 8, pp. 610-622, 2007.

[121] A. Hirano and T. Matsui, "Vascular structures in brain tumors," Human Pathology, vol. 6, no. 5, pp. 611-621, 1975.

[122] F. Winkler, Y. Kienast, M. Fuhrmann et al., "Imaging glioma cell invasion in vivo reveals mechanisms of dissemination and peritumoral angiogenesis," Glia, vol. 57, no. 12, pp. 1306-1315, 2009.

[123] J. Holash, P. C. Maisonpierre, D. Compton et al., "Vessel cooption, regression, and growth in tumors mediated by angiopoietins and VEGF,' Science, vol. 284, no. 5422, pp. 19941998, 1999.

[124] S. Watkins, S. Robel, I. F. Kimbrough, S. M. Robert, G. EllisDavies, and H. Sontheimer, "Disruption of astrocyte-vascular coupling and the blood-brain barrier by invading glioma cells," Nature Communications, vol. 5, article 4196, 2014.

[125] R. G. W. Verhaak, K. A. Hoadley, E. Purdom et al., "Integrated genomic analysis identifies clinically relevant subtypes of glioblastoma characterized by abnormalities in PDGFRA, IDH1, EGFR, and NF1," Cancer Cell, vol. 17, no. 1, pp. 98-110, 2010.

[126] H. S. Phillips, S. Kharbanda, R. Chen et al., "Molecular subclasses of high-grade glioma predict prognosis, delineate a pattern of disease progression, and resemble stages in neurogenesis," Cancer Cell, vol. 9, no. 3, pp. 157-173, 2006.

[127] Y. Zhu, P. Ghosh, P. Charnay, D. K. Burns, and L. F. Parada, "Neurofibromas in NF1: Schwann cell origin and role of tumor environment," Science, vol. 296, no. 5569, pp. 920-922, 2002.

[128] H. Colman, L. Zhang, E. P. Sulman et al., "A multigene predictor of outcome in glioblastoma," Neuro-Oncology, vol. 12, no. 1, pp. 49-57, 2010.

[129] C. E. Pelloski, A. Mahajan, M. Maor et al., "YKL-40 expression is associated with poorer response to radiation and shorter overall survival in glioblastoma," Clinical Cancer Research, vol. 11, no. 9, pp. 3326-3334, 2005.

[130] M. S. Carro, W. K. Lim, M. J. Alvarez et al., "The transcriptional network for mesenchymal transformation of brain tumours," Nature, vol. 463, no. 7279, pp. 318-325, 2010.

[131] K. P. L. Bhat, K. L. Salazar, V. Balasubramaniyan et al., "The transcriptional coactivator TAZ regulates mesenchymal differentiation in malignant glioma," Genes and Development, vol. 25, no. 24, pp. 2594-2609, 2011.

[132] K. P. Bhat, V. Balasubramaniyan, B. Vaillant et al., "Mesenchymal differentiation mediated by NF-kappaB promotes radiation resistance in glioblastoma," Cancer Cell, vol. 24, no. 3, pp. 331346, 2013.

[133] J. V. Joseph, V. Balasubramaniyan, A. Walenkamp, and F. A. E. Kruyt, "TGF-beta as a therapeutic target in high grade gliomas-promises and challenges," Biochemical Pharmacology, vol. 85 , no. 4, pp. 478-485, 2013. 
[134] J. V. Joseph, S. Conroy, T. Tomar et al., “TGF- $\beta$ is an inducer of ZEB1-dependent mesenchymal transdifferentiation in glioblastoma that is associated with tumor invasion," Cell Death and Disease, vol. 5, no. 10, article e1443, 2014.

[135] J. V. Joseph, S. Conroy, K. Pavlov et al., "Hypoxia enhances migration and invasion in glioblastoma by promoting a mesenchymal shift mediated by the HIFlalpha-ZEB1 axis," Cancer Letters, vol. 359, no. 1, pp. 107-116, 2015.

[136] M. C. Elias, K. R. Tozer, J. R. Silber et al., "TWIST is expressed in human gliomas and promotes invasion," Neoplasia, vol. 7, no. 9, pp. 824-837, 2005.

[137] F. J. N. Motta, E. T. Valera, A. K. B. Lucio-Eterovic et al., "Differential expression of E-cadherin gene in human neuroepithelial tumors," Genetics and Molecular Research, vol. 7, no. 2, pp. 295304, 2008.

[138] S.-P. Han, J.-H. Kim, M.-E. Han et al., "SNAI1 is involved in the proliferation and migration of glioblastoma cells," Cellular and Molecular Neurobiology, vol. 31, no. 3, pp. 489-496, 2011.

[139] B. Lv, X. Yang, S. Lv et al., "CXCR4 signaling induced epithelialmesenchymal transition by PI3K/AKT and ERK pathways in glioblastoma," Molecular Neurobiology, 2014.

[140] A. Liao, R. Shi, Y. Jiang et al., "SDF-1/CXCR4 axis regulates cell cycle progression and epithelial-mesenchymal transition via up-regulation of survivin in glioblastoma," Molecular Neurobiology, 2014.

[141] P. Dahan, J. Martinez Gala, C. Delmas et al., "Ionizing radiations sustain glioblastoma cell dedifferentiation to a stem-like phenotype through survivin: possible involvement in radioresistance," Cell Death and Disease, vol. 5, Article ID e1543, 2014.

[142] E. E. Bar, A. Lin, V. Mahairaki, W. Matsui, and C. G. Eberhart, "Hypoxia increases the expression of stem-cell markers and promotes clonogenicity in glioblastoma neurospheres," American Journal of Pathology, vol. 177, no. 3, pp. 1491-1502, 2010.

[143] Y. Piao, J. Liang, L. Holmes, V. Henry, E. Sulman, and J. F. de Groot, "Acquired resistance to anti-VEGF therapy in glioblastoma is associated with a mesenchymal transition," Clinical Cancer Research, vol. 19, no. 16, pp. 4392-4403, 2013.

[144] A. Sottoriva, I. Spiteri, S. G. M. Piccirillo et al., "Intratumor heterogeneity in human glioblastoma reflects cancer evolutionary dynamics," Proceedings of the National Academy of Sciences of the United States of America, vol. 110, no. 10, pp. 4009-4014, 2013.

[145] T. Ozawa, M. Riester, Y. Cheng et al., "Most human non-GCIMP glioblastoma subtypes evolve from a common proneural-like precursor glioma," Cancer Cell, vol. 26, no. 2, pp. 288-300, 2014.

[146] A. Balbous, U. Cortes, K. Guilloteau et al., "A mesenchymal glioma stem cell profile is related to clinical outcome," Oncogenesis, vol. 3, no. 3, article e91, 2014.

[147] C. Muller, J. Holtschmidt, M. Auer et al., "Hematogenous dissemination of glioblastoma multiforme," Science Translational Medicine, vol. 6, no. 247, Article ID 247ra101, 2014.

[148] K. M. MacArthur, G. D. Kao, S. Chandrasekaran et al., "Detection of brain tumor cells in the peripheral blood by a telomerase promoter-based assay," Cancer Research, vol. 74, no. 8, pp. 21522159, 2014.

[149] J. Song, C. Wu, E. Korpos et al., "Focal MMP-2 and MMP9 activity at the blood-brain barrier promotes chemokineinduced leukocyte migration," Cell Reports, vol. 10, no. 7, pp. 1040-1054, 2015.

[150] C. P. Hofstetter, J.-K. Burkhardt, B. J. Shin et al., "Protein phosphatase 2A mediates dormancy of glioblastoma multiformederived tumor stem-like cells during hypoxia," PLoS ONE, vol. 7, no. 1, Article ID e30059, 2012.
[151] G. A. Alexiou, G. Vartholomatos, A. Karamoutsios, A. Batistatou, A. P. Kyritsis, and S. Voulgaris, "Circulating progenitor cells: a comparison of patients with glioblastoma or meningioma," Acta Neurologica Belgica, vol. 113, no. 1, pp. 7-11, 2013.

[152] L. Ricci-Vitiani, R. Pallini, M. Biffoni et al., "Tumour vascularization via endothelial differentiation of glioblastoma stem-like cells," Nature, vol. 468, no. 7325, pp. 824-830, 2010. 

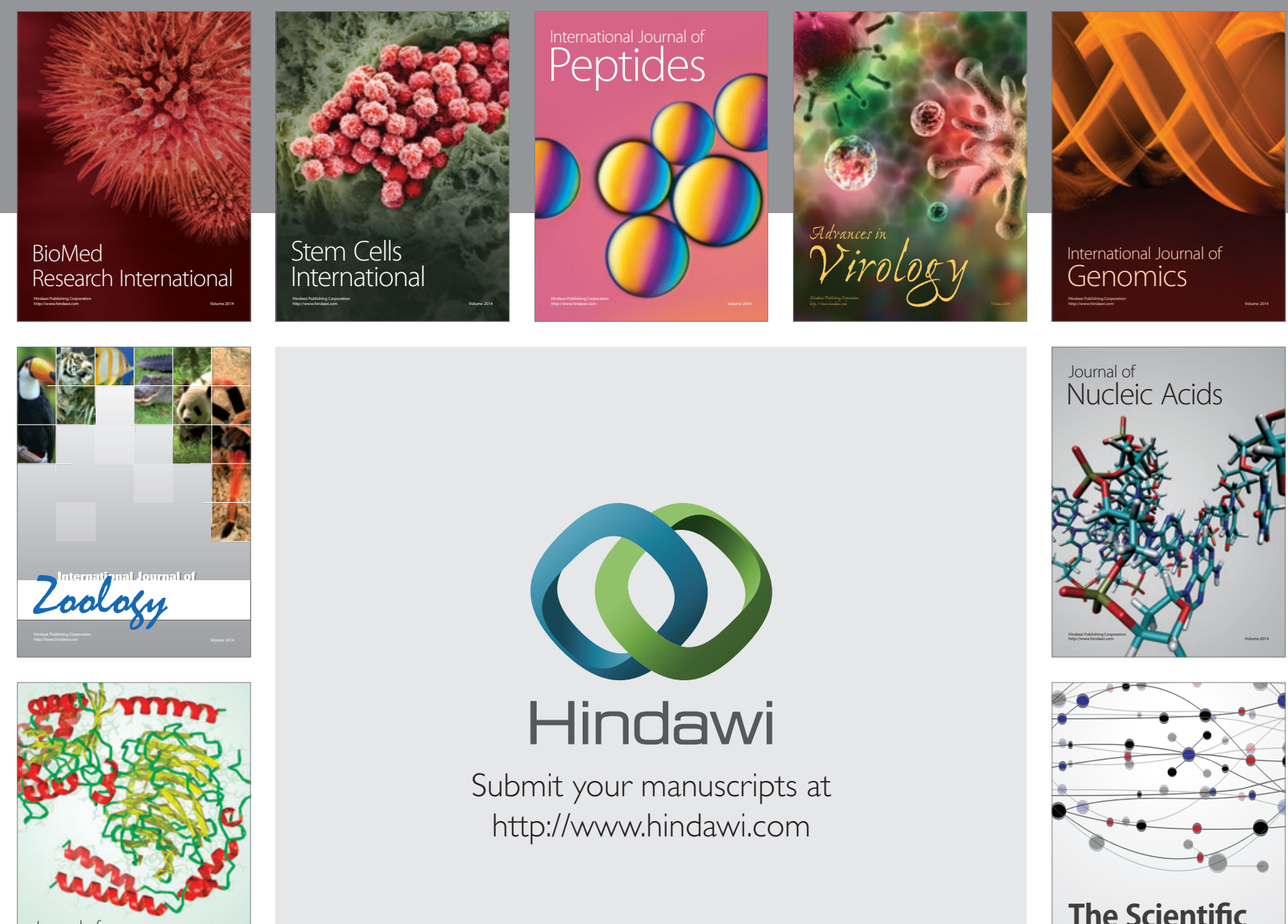

Submit your manuscripts at

http://www.hindawi.com

Journal of
Signal Transduction
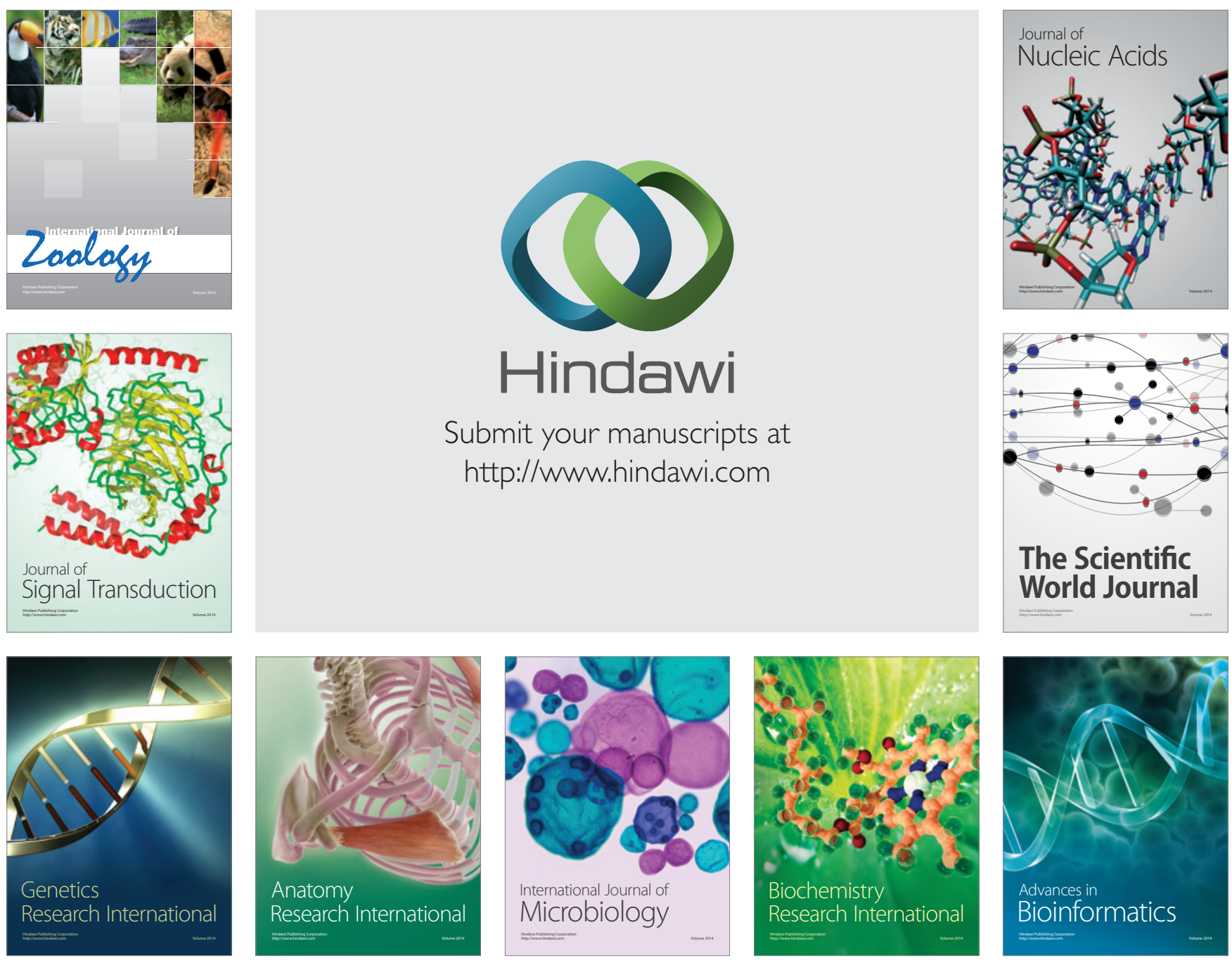

The Scientific World Journal
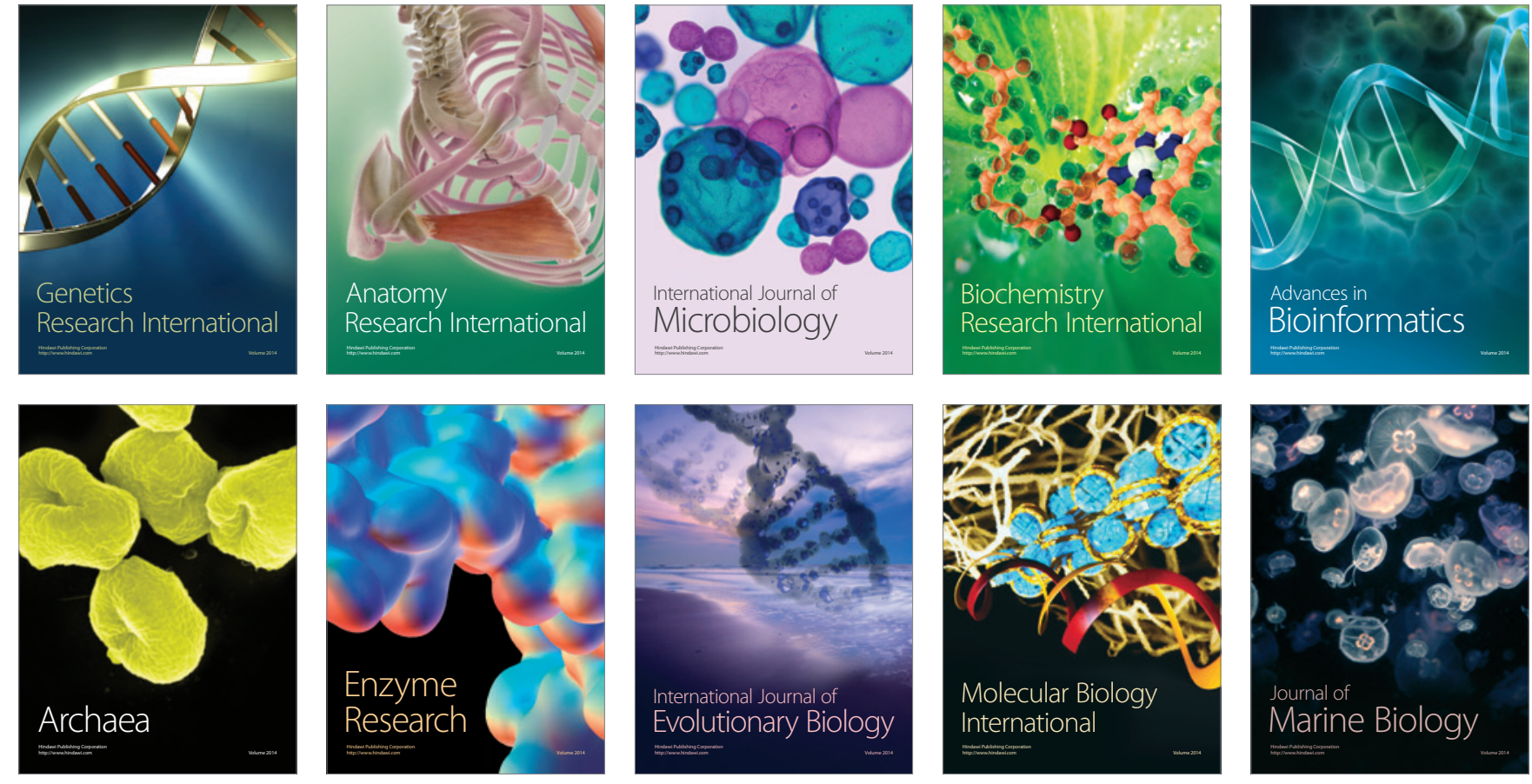